\title{
Sources of Error in Office Blood Pressure Measurement
}

\author{
Roy N. Morcos, MD, FAAFP, Kimbroe J. Carter, MD, Frank Castro, MS, \\ Sumira Koirala, MD, Deepti Sharma, MD, and Haroon Syed, MD
}

Purposes: To evaluate 2 commonly overlooked sources of error in measuring blood pressure (BP) in the office, improper patient positioning and frequency of terminal digit bias (TDB) using manual and automated (BP) devices.

Methods: BPs recorded by 3 nurses using manual and automated devices were analyzed for TDB. In the next part of the study, 294 patients were recruited and tested with each patient's BP measured twice in the table position and compared with BP measured in the chair position. To eliminate concern for position sequence, a randomized controlled trial was initially conducted.

Results: Significant TDB for the digit zero was identified in BPs measured by all nurses using a manual device. No such bias was identified for any nurse when measuring BP with an automated device. For the positional study, the randomized controlled study showed no significant sequencing effect therefore the sequence of table then chair BP measurements was adopted. Significant BP lowering was observed in 128 patients $(42.7 \%)$ in the chair compared with the table position. Misclassification of prehypertension and hypertension would have occurred in $15.3 \%$ and $16 \%$ of patients, respectively, when BP was recorded in the table instead of the chair position.

Conclusions: Significant TDB was identified for all nurses when using a manual but not an automated device. Patient positioning on the examination table resulted in elevations of systolic and diastolic BPs. (J Am Board Fam Med 2019;32:732-738.)

Keywords: Blood Pressure, Hypertension, Patient Positioning, Prehypertension

The prevalence of hypertension is increasing in the United States and worldwide, causing a significant burden of disease. ${ }^{1}$ Hypertension doubles the risk of coronary heart disease, congestive heart failure, stroke, chronic kidney disease, and peripheral arterial disease. Among all risk factors, hypertension

This article was externally peer reviewed.

Submitted 8 March 2019; revised 1 May 2019; accepted 6 May 2019.

From the Department of Family and Community Medicine, Northeast Ohio Medical University, Rootstown, OH (RNM, SK); Department of Family Medicine Residency Program, St. Elizabeth Boardman, Mercy Health (RNM); Medical Decision Making Society of Youngstown Ohio, c/o Jeghers Medical Index, St. Elizabeth Youngstown Hospital, Youngstown, $\mathrm{OH}$ (FC, KJC); Family Medicine Residency Program, St. Elizabeth Boardman, Mercy Health (SK, DS); Department of Pathology, Northeast Ohio Medical University, Rootstown, OH (KJC).

Funding: This study was funded by Medical Research Committee Grant 17-015.

Conflict of interest: none declared.

Corresponding author: Roy N. Morcos, MD, St. Elizabeth Boardman Family Health Center, 8423 Market St., Ste. 101, Boardman, OH 44512 (E-mail: Roy_Morcos@mercy.com). ranks first in disability-adjusted life-years worldwide. ${ }^{2,3}$ The diagnosis of hypertension is based on indirect measurements of blood pressure (BP) using office, ambulatory, or home $\mathrm{BP}$ devices. Although the office BP measurement is not ideal, it is most commonly used to diagnose and monitor patients' responses to therapy. Most published trials of treatment recommendations are based on office BP measurements. ${ }^{4,5}$

BP measurements are subject to errors such as terminal digit bias (TDB) - an observer's preference for a last digit, usually zero, and a tendency to round up or down the BP measurement to that digit. ${ }^{7,8}$ Another source of error is inappropriate patient positioning (eg, having the patient sit on an examination table instead of in a comfortable chair). An observational study ${ }^{9}$ revealed that the recommended patient positioning in a chair was followed in only 10 of 25 primary care offices, and in the remaining, patients were seated on the examination table. A 5-minute rest period before measuring $\mathrm{BP}$ was allowed in only 10 of 
25 offices, and an automated device was utilized in only 2 of 25 offices.

In a previous study using a manual (aneroid) device, we showed that such improper positioning resulted in misclassification of prehypertension and hypertension in $7.4 \%$ and $5.9 \%$ of patients, respectively. ${ }^{9}$ Although the automated device is the preferred BP measurement method, manual aneroid devices continue to be used in medical offices. ${ }^{8-12}$ Accurate and precise office BP measurement with manual or automated devices is essential to adequately diagnose and treat hypertension.

Proper patient positioning is emphasized in the medical literature, but we could not find the impact improper positioning has on misclassification of patients as having prehypertension or hypertension. The article studies 2 commonly overlooked sources of error in measuring BP in the office-the impact of improper patient positioning and frequency of TDB. The accuracy and reliability of such measurements is of utmost importance to provide the best possible patient care.

\section{Methods}

\section{Terminal Digit Bias (TDB)}

Observational Study

BPs recorded by 3 nurses using manual (aneroid) and automated devices were analyzed for TDB. BP measurements with manual and automated devices were obtained from patient charts and categorized by observer and type of device used. The manual device used in the study was the Welch Allyn CE0297 aneroid sphygmomanometer. The automated device was the Omron Digital BP Monitor, Model HEM-907 XL, which has been certified and used in several major hypertension studies. ${ }^{12}$ Overall, 3000 BP terminal digit observations were evaluated. There were 250 systolic and 250 diastolic observations obtained by each of the 3 nurses using manual and automated devices. The frequency of terminal digits was calculated for both sets of data.

\section{Statistical Analysis}

Manual BP observations having terminal digits 0,2 , 4,6 , and 8 were analyzed using the $\chi^{2}$ test for independence with 4 degrees of freedom. Automated BP observations having terminal digits 0 through 9 were analyzed using the $\chi^{2}$ test for independence with 9 degrees of freedom. $\chi^{2}$ tests were performed in Microsoft Excel for each nurse with $P<.01$ considered statistically significant.

\section{Effects of Patient Positioning on BP Measurements} Randomized controlled trial

A randomized controlled trial (ClinicalTrials.gov Identifier: NCT03460249) was initially conducted to evaluate the effect of sequence of patient positioning on BP measurements, that is, the sequence of table followed by chair BP measurements versus chair followed by table BP measurements. Thirty patients were randomized, and the results were analyzed with $\chi^{2}$ tests, finding no difference in the sequence. As a result, a table to chair sequence was adopted for the study.

\section{Standard Deviation Estimate}

To estimate the standard deviation of the automated device and observer, the BP of a healthy, nonhypertensive individual was repeatedly measured in the standard seated position. One hundred twenty BP measurements were obtained by the same observer over 2 days to minimize subject and operator fatigue. For the chair and table positions, the systolic BP standard deviations were $4.20 \mathrm{~mm}$ $\mathrm{Hg}$ and $4.33 \mathrm{~mm} \mathrm{Hg}$, respectively, and the diastolic BP standard deviations were $3.62 \mathrm{~mm} \mathrm{Hg}$ and 4.26 $\mathrm{mm} \mathrm{Hg}$, respectively.

\section{Patients}

The study population consisted of patients who consecutively presented to a teaching family medicine center for a scheduled appointment. Adult patients, aged 18 years and older, were informed about the study and invited to participate. Exclusion criteria were patients who declined participation for any reason, those in significant pain or distress who may have been unable to complete the protocol, and those with limited mobility who may have had difficulty getting up to the examination table. The study was approved by a regional institutional review board for human subjects, and informed consent was obtained from the patients. There were a total of $1176 \mathrm{BP}$ measurements from 294 patients. Of the 294 participants, 188 (63.9\%) were female, 58 (19.7\%) were diabetic, 141 (48.0\%) were hypertensive, 106 (36.1\%) were hyperlipidemic, $36(12.2 \%)$ had cardiovascular disease, and $158(53.7 \%)$ were current or formerly smokers. The median age was 50.5 years with the youngest being 18 years and the oldest, 90 years. 


\section{Data Collection}

Four BP readings were obtained for each individual using an automated device, 2 in the table position followed by 2 in the chair position. BPs were measured with an automated device for most patients. Overall, 294 individuals agreed to participate and were tested. A manual device was used for patients with significant arrhythmia, such as atrial fibrillation and tachycardia, or if a measurement could not be obtained with the automated device. ${ }^{13}$ The guidelines of the American Heart Association (AHA) for patient positioning and BP measurement technique were followed. ${ }^{14}$ Before beginning the study, detailed instructions regarding proper $\mathrm{BP}$ measurement technique were provided to the nurses who obtained the data. A medical chart review was performed for all patients, and clinical data were obtained including age, sex, smoking status and presence of diabetes, cardiovascular disease, hypertension, and hyperlipidemia. Other than age, all collected data were binary, categorized as present or absent.

\section{Classification of Hypertension}

In accordance with the commonly used Seventh Report of the Joint National Committee on Prevention, Detection, Evaluation, and Treatment of High Blood Pressure (JNC-7) guideline definitions, a normal $\mathrm{BP}$ is below 120/80 $\mathrm{mm} \mathrm{Hg}$, prehypertension is a $\mathrm{BP}$ between 120 to 139 and 80 to $89 \mathrm{~mm} \mathrm{Hg}$, and hypertension is a $\mathrm{BP}$ equal to or above $140 / 90 \mathrm{~mm} \mathrm{Hg} .{ }^{4}$ The average systolic and diastolic BP values in the 2 positions were calculated for each patient's readings, and the patient was classified as having normal $\mathrm{BP}$, prehypertension, or hypertension. Classification was repeated using another guideline published by the American College of Cardiology (ACC)/ AHA in 2017, in which normal BP is defined as below 120/80 $\mathrm{mm} \mathrm{Hg}$, elevated $\mathrm{BP}$ as 120 to 129 and below $80 \mathrm{~mm} \mathrm{Hg}$, and hypertension as 130/80 mm Hg or above. ${ }^{12}$ Patients whose BPs showed significant lowering between average table and chair readings resulting in a change of classification from prehypertension (elevated BP) to normal or from hypertension to either prehypertension or normal were considered misclassified. For example, a patient with a table average reading of $128 / 87 \mathrm{~mm} \mathrm{Hg}$ and a chair average reading of $118 / 78 \mathrm{~mm} \mathrm{Hg}$ would be misclassified as having prehypertension according to the JNC-7 guideline.

\section{Statistical Analysis}

The difference between the average BP values from the table and chair positions was calculated for each patient's systolic and diastolic readings, and an unpaired $t$-test performed to analyze the data. The standard error of the mean of these differences was determined and a 2 -sided $95 \%$ confidence upper bound for the standard error of the difference $\left(S E_{d}\right)$ was based on the normal distribution's upper bound of $1.96 \times S E_{d}$. Using the normal distribution assumption, this results in upper bounds of $8.36 \mathrm{~mm}$ $\mathrm{Hg}$ for systolic BP and $7.74 \mathrm{~mm} \mathrm{Hg}$ for diastolic BP. Patient observed differences were compared against the confidence upper bound to identify significant systolic and diastolic BP changes due to patient positioning. For example, consider a patient with 2 systolic automated $\mathrm{BP}$ readings in the table position averaging $129 \mathrm{~mm} \mathrm{Hg}$ and 2 systolic automated BP readings in the chair position averaging $119 \mathrm{~mm}$ $\mathrm{Hg}$. Assuming an $S E_{d}$ of $4.26 \mathrm{~mm} \mathrm{Hg}$ and an upper bound of $8.36 \mathrm{~mm} \mathrm{Hg}$, the observed $10 \mathrm{~mm} \mathrm{Hg}$ difference in average systolic BP is considered significant since the degree of random variation from the device and observer is not expected to exceed $8.36 \mathrm{~mm} \mathrm{Hg}$ for systolic BP.

Data from the observational study were processed using the Binary Logistic and Probit Regression function with significant BP lowering as the dependent variable. Clinical factors identified in the data set were used as independent variables. Logistic regression was performed using the Excel Add-in Real Statistics Resource Pack software (Release 4.3, www.real-statistics.com) to determine if any of these clinical factors could predict significant lowering of $\mathrm{BP}$ due to change in patient positioning. We also evaluated misclassification of hypertensive disease according to clinical data subgroups.

\section{Results}

\section{Terminal Digit Bias}

Table 1 presents the distribution of terminal digits for 1 of the nurses using a manual device. In the absence of TDB, the predicted prevalence of each terminal digit is 150 and expected to be equally distributed. In this example, 350 measurements ended with a zero, indicating highly significant bias for that digit $(P<.01)$. A preference for the ter- 
Table 1. Example Distribution of Terminal Digits for One Nurse Using a Manual Device

\begin{tabular}{lcc}
\hline Terminal Digit & Actual & Expected $^{*}$ \\
\hline 0 & 350 & 150 \\
2 & 130 & 150 \\
4 & 73 & 150 \\
6 & 92 & 150 \\
8 & 105 & 150 \\
Total & 750 & 750 \\
\hline
\end{tabular}

$P<.01$.

${ }^{*}$ Expected number in the absence of terminal digit bias.

minal digit zero was found for each of the 3 nurses when measuring BP with a manual device, Table 2. The degree of TDB varied between the nurses but was highly significant for each $(P<.01)$. No such TDB was found for any of the nurses when BP was measured with the automated device.

\section{Effect of Patient Positioning on BP Measurements}

The outcomes of the randomized controlled trial showed that the sequence of patient positioning (table to chair position followed by chair to table position vs chair to table position followed by table to chair position) did not affect the differences in BP. The results of the $\chi^{2}$ test for independence with 1 degree of freedom were 0.37 for systolic BP and 1.00 for diastolic BP. These findings were not significant for the positional effect at a critical value of 3.84 , representing $95 \%$ probability of no difference. Thus, the sequence of table position first followed by chair position was adhered to in the subsequent part of the study.

The results of BP measurements comparing table and chair positions are summarized in Table 3. The BP was significantly lower in the chair position compared with the table position in 128 individuals (43.5\%). Compared with BP measurements in the table position, 46 patients (15.6\%) would have been misclassified with prehypertension, and 48 patients
(16.3\%) would have been misclassified with hypertension, based on the JNC-7 definition.

Based on the ACC/AHA guideline, $4.8 \%$ of patients would have been misclassified as having elevated $\mathrm{BP}$, and $20.1 \%$ of patients would have been misclassified as having hypertension (Table 3). Logistic regression analysis of age, sex, the presence of diabetes, cardiovascular disease, hypertension, hyperlipidemia, and smoking showed that these independent factors did not predict significant diastolic BP lowering with change in patient positioning (Table 4). Interestingly, cardiovascular disease was associated with more systolic BP lowering while hyperlipidemia was associated with less systolic BP lowering.

\section{Discussion}

An accurate and reliable BP measurement is essential for diagnosing and managing hypertension. The family physician is ideally positioned to identify an early BP rise in asymptomatic individuals and can have a major influence on reducing hypertension-related morbidities. To achieve this goal, meticulous attention to the BP measurement technique and instruments must be followed. Sources of error may be due to the equipment used or to the individual measuring the BP. In this study, we have examined 2 such sources of error that family physicians are likely to encounter. The importance of minimizing errors is confirmed in a large metaanalysis showing that a decrease in systolic BP by $10 \mathrm{~mm} \mathrm{Hg}$ results in significant reduction in the risk of coronary artery disease, stroke, and heart failure. ${ }^{5}$ Another large study by Greiver ${ }^{6}$ showed that TDB decreased from $26.6 \%$ to $15.4 \%$ since the acquisition of automated devices, and patients in sites with a high level of TDB had a higher frequency of strokes, acute myocardial infarction, and angina. This highlights the relevance of TDB and the clinical importance of minimizing or eliminating it. This study confirms and expands on

Table 2. $P$-Values for the $\chi^{2}$ Test of Equality of the Proportion of Blood Pressure Ending Digits

\begin{tabular}{lcccc}
\hline & Manual Systolic & Manual Diastolic & Auto Systolic & Auto Diastolic \\
\hline Nurse 1 & Significant & Significant & Not Significant & Not Significant \\
Nurse 2 & Significant & Significant & Not Significant & Not Significant \\
Nurse 3 & Significant & Significant & Not Significant & Not Significant \\
\hline
\end{tabular}

Significant, $P<.01$.

Not Significant, $P \geq .1$. 
Table 3. Significant Blood Pressure Differences and Misclassification of Hypertensive Disease Using JNC-7 Guideline and ACC/AHA 2017 Guidelines

\begin{tabular}{|c|c|c|c|}
\hline \multicolumn{4}{|c|}{ Using JNC-7 Guideline Definition of Hypertension } \\
\hline $\mathrm{N}=294$ & Significant lowering & Misclassification, pre-hypertension & Misclassification, hypertension \\
\hline Patients, n (\%) & $128(43.5 \%)$ & $46(15.6 \%)$ & $48(16.3 \%)$ \\
\hline Systolic reading & $101(34.3 \%)$ & $24(8.2 \%)$ & $28(9.5 \%)$ \\
\hline Diastolic reading & $75(25.2 \%)$ & $27(9.2 \%)$ & $24(8.2 \%)$ \\
\hline \multicolumn{4}{|c|}{ ACC/AHA 2017 Guideline Definition of Hypertension } \\
\hline $\mathrm{N}=294$ & Significant lowering & Misclassification, elevated & Misclassification, hypertension \\
\hline Patients, n (\%) & $128(43.5 \%)$ & $14(4.8 \%)$ & $59(20.1 \%)$ \\
\hline Systolic reading & $101(34.3 \%)$ & $14(4.8 \%)$ & $27(9.2 \%)$ \\
\hline Diastolic reading & $75(25.2 \%)$ & $0(0.0 \%)$ & $41(16.9 \%)$ \\
\hline
\end{tabular}

ACC, American College of Cardiology; AHA, American Heart Association; JNC-7, Seventh Report of the Joint National Committee on Prevention, Detection, Evaluation, and Treatment of High Blood Pressure.

previously reported findings regarding the behavior and limitations of BP measurements. ${ }^{12}$ First, we identified a highly significant TDB for all 3 nurses tested using a manual device. There was a bias for the number zero as a terminal digit. No such bias was identified with any of the same 3 nurses using an automated device. This TDB was previously described in numerous other studies, ${ }^{19,21-29}$ most of which show that TDB is reduced but not completely eliminated by the introduction of automated devices in measuring BP. Myers and Campbell $^{11}$ found evidence of TDB of $14 \%$ of readings when using the BpTRU automated device, when the expected proportion of zero terminal digits is $10 \%$. In another study, no TDB was identified when BP were measured with a BpTRU device although the actual data are not shown. ${ }^{31}$ It is important to note that not all automated devices are necessarily similar since the BP is not directly measured but calculated based on a proprietary

Table 4. Logistic Regression of the Clinical Factors Influencing the Significant Blood Pressure Difference

\begin{tabular}{lcc}
\hline Clinical Factor & Systolic $P$-Value & Diastolic $P$-Value \\
\hline Age & .78 & .29 \\
Sex & .93 & .90 \\
Hypertension & .17 & .67 \\
Diabetes & .11 & .15 \\
Hyperlipidemia & $.0046^{*}$ & .33 \\
Smoker & .93 & .59 \\
Cardiovascular disease & $.0054^{\dagger}$ & .63 \\
\hline
\end{tabular}

*Patients with hyperlipidemia had significantly decreased risk of misclassification.

${ }^{\dagger}$ Patients with cardiovascular disease had significantly increased risk of misclassification. algorithm that differs according to each manufacturer. One study by Mengden ${ }^{30}$ showed that use of automated devices minimized TDB but there was another bias in data recording because BPs were clustered around therapeutic cutoff levels. In our study, there was no evidence of TDB when BP was measured with the OMRON automated device.

We previously reported that $\mathrm{BP}$ measurements obtained with the patient sitting on the examination table rather than in a chair often results in elevated levels, which can lead to misclassification of hypertension. ${ }^{9}$ A concern was raised about the chosen sequence, table first then chair position, and whether the opposite sequence would have had the same effect. A need for randomization of the order of BP measurements was also discussed in a recent review of the implications of $\mathrm{BP}$ measurement techniques. ${ }^{10}$ Herein, we found that the sequence of BP measurements did not affect the difference in BP between the 2 positions. No previous studies have evaluated the difference in BP between table versus sitting positions and the impact of such improper positioning on misclassification of prehypertension and hypertension when $\mathrm{BP}$ is measured with an automated device. Lacruz et $\mathrm{al}^{17}$ found a significant increase in BP in the sitting versus lyingdown position.

We previously found that the chair position resulted in a significant decrease in BP compared with the table position in $30.4 \%$ of patients when using a manual device. ${ }^{9}$ In this study, we also found that the chair position resulted in a significant and even greater decrease in $\mathrm{BP}$ compared with the table position in $42.7 \%$ of patients when using an automated device. Further, we found more misclas- 
sification of prehypertension and hypertension using either the JNC-7 or ACC/AHA guidelines when the $\mathrm{BP}$ is predominantly measured with the automated compared with the manual method. The reasons for these differences between devices are not known but may result from another type of observer bias. When using a manual device, an observer's knowledge of the BP initially measured in the table position may affect the BP reading in subsequent measurements. This is an example of anchoring bias, ${ }^{16}$ which is not expected to occur with an automated device. Further studies exclusively using automated devices are needed to confirm the lack of anchoring bias. Furthermore, highly significant TDB was identified with the use of a manual but not with an automated device.

\section{Limitations}

A weakness of our study is that BP was measured only twice in each position. Other studies have obtained 3 or more measurements to ensure a stable and reliable BP level. ${ }^{13,17}$ However, in a recent study of BP measurements and mortality, only 2 measurements were obtained, and an average was calculated. ${ }^{18}$ We have similarly chosen to obtain only 2 BP readings to better simulate real-life conditions that are likely to be encountered in a busy primary care practice.

\section{Conclusions}

There are numerous national and international guidelines about BP thresholds for diagnosing hypertension, but regardless of the definitions used, it is essential to accurately and reproducibly obtain BP measurements. The commonly accepted method of choice for BP measurement is ambulatory 24-hour monitoring. ${ }^{14,15}$ It is however used mostly in research, and its implementation to the general population remains challenging because of equipment cost and other difficulties. Office measurements remain the most widely adopted method and are often supplemented by home BP monitoring. ${ }^{19}$ These results confirm the importance of proper patient positioning in a comfortable chair when measuring BP. Further, TDB occurs with manual but not with the automated device we used, thus confirming the potential advantage of automated devices in obtaining an accurate and reliable office BP measurement. ${ }^{19}$ Anchoring bias may also occur with repeated manual $\mathrm{BP}$ measurements, adding to the uncertainty of such measurements.

We wish to thank the nursing staff at St. Elizabeth Boardman Family Medicine Center for the data collection and the St. Elizabeth Youngstown Hospital Medical Library for literature search and retrieval.

To see this article online, please go to: http://jabfm.org/content/ 32/5/732.full.

\section{References}

1. Kearney PM, Whelton M, Reynolds K, Muntner P, Whelton PK, He J. Global burden of hypertension: analysis of worldwide data. Lancet 2005;365:217-23.

2. Murray CJ, Lopez AD. Measuring the global burden of disease. N Engl J Med 2013;369:448-57.

3. Lim SS, Vos T, Flaxman AD, et al. A comparative risk assessment of burden of disease and injury attributable to 67 risk factors and risk factor clusters in 21 regions, 1990-2010: a systematic analysis for the Global Burden of Disease Study 2010. Lancet 2012; 380:2224-60.

4. Chobanian AV, Bakris GL, Black HR, et al. The Seventh Report of the Joint National Committee on Prevention, Detection, Evaluation, and Treatment of High Blood Pressure: the JNC 7 report. JAMA 2003;289:2560-72.

5. Ettehad D, Emdin CA, Kiran A, et al. Blood pressure lowering for prevention of cardiovascular disease and death: a systematic review and meta-analysis. Lancet 2016;387:957-67.

6. Greiver M, Kalia S, Voruganti T, et al. Trends in end digit preference for blood pressure and associations with cardiovascular outcomes in Canadian and UK primary care: a retrospective observational study. BMJ Open 2019;9:e024970.

7. Bailey RH, Bauer JH. A review of common errors in the indirect measurement of blood pressure. Sphygmomanometry. Arch Intern Med 1993;153:2741-8.

8. Nietert PJ, Wessell AM, Feifer C, Ornstein SM. Effect of terminal digit preference on blood pressure measurement and treatment in primary care. Am J Hypertens 2006;19:147-52.

9. Morcos RN, Carter KJ, Castro F, et al. Getting the hypertension Dx right: patient positioning matters. J Fam Pract 2018;67:199;201:206-7.

10. Drawz P. Clinical implications of different blood pressure measurement techniques. Curr Hypertens Rep 2017;19:54.

11. Myers MG, Campbell NR. Unfounded concerns about the use of automated office blood pressure measurement in SPRINT. J Am Soc Hypertens 2016;10:903-5.

12. Myers MG, Godwin M, Dawes M, Kiss A, Tobe SW, Kaczorowski J. Measurement of blood pressure 
in the office: recognizing the problem and proposing the solution. Hypertension 2010;55:195-200.

13. SPRINT Research Group, Wright JT Jr, Williamson JD, et al. A randomized trial of intensive versus standard blood-pressure control. N Engl J Med 2015;373:2103-16.

14. Staessen JA, Li Y, Hara A, Asayama K, Dolan E, O'Brien E. Blood pressure measurement Anno 2016. Am J Hypertens 2017;30:453-63.

15. Pickering TG, Hall JE, Appel LJ, Falkner BE, et al. Recommendations for blood pressure measurement in humans and experimental animals: Part 1: blood pressure measurement in humans: a statement for professionals from the Subcommittee of Professional and Public Education of the American Heart Association Council on High Blood Pressure Research. Hypertension 2005;45:142-61.

16. Saposnik G, Redelmeier D, Ruff CC, Tobler PN. Cognitive biases associated with medical decision: a systematic review. BMC Med Inform Decis Mak 2016;16:138.

17. Lacruz ME, Kluttig A, Kuss O, et al. Short-term blood pressure variability-variation between arm side, body position and successive measurements: a population-based cohort study. BMC Cardiovasc Disord 2017;17:31.

18. Banegas JR, Ruilope LM, de la Sierra A, et al. Relationship between clinic and ambulatory blood-pressure measurements and mortality. N Engl J Med 2018;378:1509-20.

19. Myers MG. Replacing manual sphygmomanometers with automated blood pressure measurement in routine clinical practice. Clin Exp Pharmacol Physiol 2014;41:46-53.

20. Thavarajah S, White WB, Mansoor GA. Terminal digit bias in a specialty hypertension faculty practice. J Hum Hypertens 2003;17:819-22.

21. Harrison WN, Lancashire RJ, Marshall TP. Variation in recorded blood pressure terminal digit bias in general practice. J Hum Hypertens 2008;22:163-7.
22. Alsanjari ON, de Lusignan S, van Vlymen J, et al. Trends and transient change in end-digit preference in blood pressure recording: studies of sequential and longitudinal collected primary care data. Int J Clin Pract 2012;66:37-43.

23. Ayodele OE, Akinyemi SO, Adeniji AO, et al. Prevalence of end-digit preference in recorded blood pressure by nurses: a comparison of measurements taken by mercury and electronic blood pressuremeasuring devices. S Afr Fam Pract 2013;55:73-7.

24. Boonyasai R, Carson KA, Marsteller JA, et al. A bundled quality improvement program to standardize clinical blood pressure measurement in primary care. J Clin Hypertens 2018;20:324-33.

25. Cloutier L, Schiffrin EL. Hypertension prevalence and control: impact of method of blood pressure measurement. Curr Cardiovasc Risk Rep 2012;6: 267-73.

26. McManus RJ, Mant J, Hull MR, et al. Does changing from mercury to electronic blood pressure measurement influence recorded blood pressure? An observational study. Br J Gen Pract 2003;53:953-6.

27. Nargesi AA, Ghazizadeh Z, Larry M, et al. Manual or automated sphygmomanometer? A historical cohort to quantify measurement bias in blood pressure recording. J Clin Hypertens 2014;16:716-721.

28. Parker RA, Paterson M, Padfield P, et al. Are selfreported telemonitored blood pressure readings affected by end-digit preference: a prospective cohort study in Scotland. BMJ Open 2018;8:e19431.

29. Rinfret F, Cloutier L, Wistaff R, et al. Comparison of different automated office blood pressure measurement devices: evidence of nonequivalence and clinical implications. Can J Cardiol 2017;33:1639-44.

30. Mengden T, Asmar R, Kandra A, et al. Use of automated blood pressure measurements in clinical trials and registration studies: data from the VALTOP Study. Blood Press Monit 2010;15:188-94.

31. Campbell N, Conradson HE, Kang J, et al. Automated assessment of blood pressure using BpTRU compared with assessments by a trained technician and a clinic nurse. Blood Press Monit 2005;10:257-62. 